\title{
Protein Recruitment through Indirect Mechanochemical Interactions
}

\author{
Andriy Goychuk and Erwin Frey॰ \\ Arnold Sommerfeld Center for Theoretical Physics and Center for NanoScience, Department of Physics, \\ Ludwig-Maximilians-Universität München, Theresienstraße 37, D-80333 Munich, Germany
}

(Received 23 May 2019; published 21 October 2019)

\begin{abstract}
Some of the key proteins essential for important cellular processes are capable of recruiting other proteins from the cytosol to phospholipid membranes. The physical basis for this cooperativity of binding is, surprisingly, still unclear. Here, we suggest a general feedback mechanism that explains cooperativity through mechanochemical coupling mediated by the mechanical properties of phospholipid membranes. Our theory predicts that protein recruitment, and therefore also protein pattern formation, involves membrane deformation and is strongly affected by membrane composition.
\end{abstract}

DOI: 10.1103/PhysRevLett.123.178101

Protein pattern formation is essential for the spatial organization of intracellular processes [1]. Examples of biological significance include Min oscillations that guide the positioning of the $Z$ ring to midcell in E. coli [2], the roles of cell polarization in determining the position of a new growth zone or bud site in S. cerevisiae [3] and the anteroposterior axis of the embryo in C. elegans [4], and spatiotemporal patterns formed by members of the Rho family of GTPases in eukaryotic cells [5]. Such selforganized patterns are the product of a dynamic interplay between diffusion (both in the cytosol and on the membrane) and biochemical reactions among proteins and between proteins and the membrane. A crucial motif in all of the biochemical reaction networks that drive these processes is a nonlinear feedback mechanism, which is generally termed recruitment. Here, membrane-bound proteins facilitate the binding of other soluble proteins from the cytosol to the membrane [1]. For example, in $E$. coli, membrane-bound MinD is said to recruit both cytosolic MinD and MinE to the membrane. What, then, is the physical basis for such cooperative binding between proteins and the membrane? One could adopt a purely chemical perspective and suggest an explanation based on classical concepts of binding cooperativity $[6,7]$. However, an indiscriminately high chemical affinity between recruiting proteins would also promote protein aggregation in the cytosol as an unwanted side effect. Then, to still facilitate specific recruitment to the membrane, a possible strategy is for individual proteins to change their conformation upon binding to the membrane so as to become chemically affine

Published by the American Physical Society under the terms of the Creative Commons Attribution 4.0 International license. Further distribution of this work must maintain attribution to the author(s) and the published article's title, journal citation, and DOI. scaffolds for other proteins [8,9]. In addition to these chemical interactions, binding of proteins to membranes inevitably invokes forces that can lead to membrane deformation.

Here, we show how such mechanochemical coupling can lead to a mechanism for the cooperative recruitment of proteins to phospholipid membranes and thereby provide an alternative strategy for cooperative membrane binding. The basic idea is very simple: Attractive forces between proteins and phospholipids facilitate protein attachment to the membrane. As equal and opposite forces must act on the membrane as well, protein binding will induce mechanical deformation of the membrane. Indeed, it is well known that membrane shape changes can be caused by curvatureinducing polymers and proteins [10-19] containing BinAmphiphysin-Rvs (BAR) domains [20-26] and-as recently shown [27] - also by the Min family of proteins. Equilibrium theories of the coupling between proteins and the membrane generally lead to membrane-mediated interactions between membrane-bound proteins, as reviewed in Refs. [28-30]. The physical origin of such interactions may be a hydrophobic mismatch for integral proteins [31-35], surface interactions that depend on curvature $[24,25,32,36-$ 41], or membrane shape fluctuations $[42,43]$. Furthermore, these interactions may also depend on the packing density [44] and composition [45,46] of the membrane. Then, proteins that are bound to the membrane effectively attract or repel each other [47-50] and form different aggregates [30,38,40,51-54]. Here, however, we do not focus on such self-organization effects. Instead, we ask a different and independent question, namely, how membrane deformations affect the affinity and kinetic (un)binding rates of proteins. We propose a general protein recruitment mechanism caused by indirect interactions facilitated through mechanical deformations of the membrane.

As we are interested in quantifying the effect of membrane-mediated interactions on the kinetic rates of 
protein membrane binding and unbinding, we need to analyze the dynamics of proteins that are subject to both cytosolic diffusion (with diffusion constant $D$ ) and a chemical potential gradient $\mu(\mathbf{x})$ caused by the mechanochemical interaction of proteins with the membrane. This is described by a Smoluchowski equation [55,56] for the cytosolic protein density $c(\mathbf{x}, t)$ :

$$
\partial_{t} c(\mathbf{x}, t)=D \nabla^{2} c(\mathbf{x}, t)+\frac{D}{k_{\mathrm{B}} T} \nabla(c(\mathbf{x}, t) \nabla \mu(\mathbf{x})) .
$$

As proteins diffuse freely in the cytosol and interact with the membrane only within some narrow range $d$, a typical spatial profile of the chemical potential is initially flat in the cytosol $(\mu=0)$ and then monotonically approaches that of the proteins at the membrane, $\mu_{\mathrm{m}}(m, \mathbf{u})=\delta F[m(\boldsymbol{\sigma}), \mathbf{u}(\boldsymbol{\sigma})] / \delta m(\boldsymbol{\sigma})$, where $F$ denotes the free energy functional describing the mechanochemical interaction between proteins and the membrane [57]. In general, $F$ will depend on both the membrane's protein density $m(\boldsymbol{\sigma})$ and its mechanical state $\mathbf{u}(\boldsymbol{\sigma})$ at position $\boldsymbol{\sigma}$ on the membrane surface; see Fig. 1 for an illustration.

The local free energy density describing the mechanochemical coupling between proteins and the membrane is determined by lipid-lipid and protein-lipid interactions. We assume that a fluid phospholipid membrane can, on a coarse-grained level, be considered as an elastically deformable thin sheet, with bulk modulus $\kappa_{\mathrm{s}}$, vanishing shear modulus, and a bending modulus $\kappa_{\mathrm{b}}$ that is equal for both principal curvatures [58]. For low levels of strain, we separate the mechanical degrees of freedom (d.o.f.) of the membrane into lateral stretching and out-of-plane bending [59] and write each mechanical contribution to the free energy as

$$
f_{\text {mech }}(u)=\frac{1}{2} \kappa\left(u-u_{0}\right)^{2} .
$$

Here, $u \in\{\rho, H\}$ is a placeholder variable for the mechanical state (conformation) of the membrane, $\kappa \in\left\{\kappa_{\mathrm{s}}, \kappa_{\mathrm{b}}\right\}$ denotes the respective membrane bulk and bending modulus, and $u_{0}$ denotes the equilibrium conformation (equilibrium density or intrinsic spontaneous curvature [60]).

As outlined above, there are several factors that determine the interaction between proteins and the membrane. Conceptually, one may distinguish between two limiting cases [Fig. 1(b)]: (A) protein anchorage through a membrane targeting domain that penetrates into the inner leaflet of the phospholipid bilayer and induces lateral membrane strain, or (B) protein attachment to the membrane by surface interactions and membrane bending. In both cases, the binding energy, $E_{\mathrm{B}} \geq E_{\text {opt }}$, of a protein to the membrane will depend on the mechanical state (conformation) of the membrane, $u$. In particular, the binding will be strongest, $E_{\mathrm{B}}=E_{\mathrm{opt}}$, for some optimal mechanical state $u_{\mathrm{opt}}$, where it attains an optimal value (a)

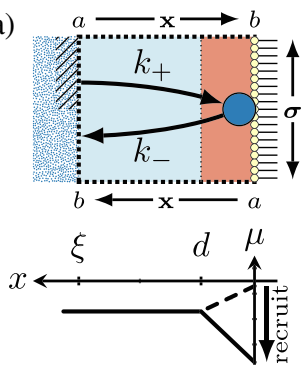

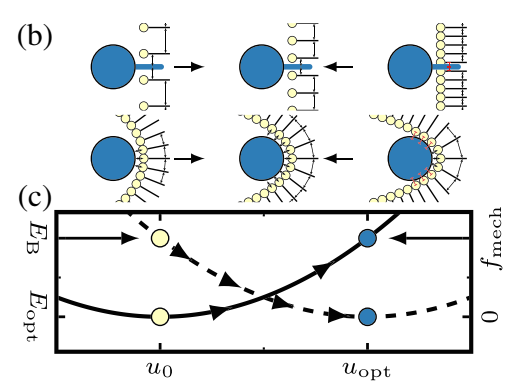

FIG. 1. (a) We divide intracellular space into reaction compartments (top), each containing one protein on average (blue dot), and identify the distance from the membrane $x$ as the reaction coordinate. The proteins diffuse freely far away from the membrane $(x>d$, blue area) and sense a chemical potential $\mu$ close to the membrane $(x<d$, red area), which facilitates protein binding. Membrane-bound proteins modulate the chemical potential $\mu$ (arrow) and therefore induce a positive feedback in the attachment rate $k_{+}$. (b) Exaggerated membrane deformation illustrates protein interactions. Attachment occurs by (top) insertion of an anchor into the inner leaflet or by (bottom) deposition through attractive surface interactions. (c) In both cases, the mechanical state change (arrows, $u \in\{\rho, H\}$ ) influences both the energy density $f_{\text {mech }}$ (solid line) stored in the deformation of the membrane and the binding energy of a protein $E_{\mathrm{B}}$ (dashed line).

$E_{\text {opt }}<0$ [Fig. 1(c)]. This optimal conformation can be understood as a compromise between maximal attractive interactions between proteins and lipids, and minimal steric repulsion [Fig. 1(b)]. As the membrane becomes crowded with proteins, the binding energy will be reduced due to protein-protein interactions [61]. Given that the repulsive part of the Lennard-Jones potential scales as $\propto r^{-12}$ at small distances $r$, this may be accounted for by a factor $1+\gamma m^{6}$, with $\gamma<0$; note that the membrane protein density scales as $m \propto r^{-2}$. Then, a Taylor expansion of the chemical free energy density to lowest order in the membrane conformation, $u$, yields

$f_{\text {chem }}(u, m)=m\left[E_{\mathrm{opt}}\left(1+\gamma m^{6}\right)+\frac{1}{2} \epsilon\left(u-u_{\mathrm{opt}}\right)^{2}\right]$,

where the parameter $\epsilon$ characterizes how strongly the membrane conformation affects protein binding. As noted above, there is a broad range of cytosolic proteins that bind to lipid membranes in a curvature-dependent manner [17,20-23,26,62]; cf. Fig. 1(b), lower panel. For example, protein-curvature coupling can arise from bending proteins to the local membrane curvature $[25,37,39,40,63-65]$ or by bending the membrane to the shape of the proteins in order to maximize attractive interactions [Fig. 1(b)]. In the following, we specifically consider proteins that couple to the membrane curvature (sum of the two principal curvatures), $u \equiv H$, and discuss lipid-density-coupling proteins in the Supplemental Material (SM) [66]. 
As mechanical d.o.f. relax much faster than protein densities, we adiabatically eliminate the mechanical d.o.f. by assuming $\partial_{u} f=0$, where $f=f_{\text {mech }}+f_{\text {chem }}$ [73]. This yields a relation between the membrane conformation $u$ and the protein density $m$ on the membrane: $u(m)=$ $u_{0}+\left(u_{\text {opt }}-u_{0}\right) m /\left(m_{\times}+m\right)$. Here, the ratio between the mechanical modulus $\kappa$ and the mechanochemical coupling parameter $\epsilon$ defines a characteristic membrane protein density: $m_{\times}=\kappa / \epsilon$. For low membrane protein density, $m<m_{\times}$, the interaction between the lipids dominates, and the mechanical state of the membrane is given by the equilibrium value $u_{0}$; cf. yellow symbols in Fig. 1(c). With an increasing number of attached proteins, the membrane gradually deforms and adopts the mechanical state that is preferred by the proteins; cf. blue symbols in Fig. 1(c). There is an interplay between a mechanical energy cost that is lowest at the relaxed state of the membrane, $u_{0}$, and a binding energy gain that is highest in the deformed state of the membrane, which is optimal for protein binding, $u_{\mathrm{opt}}$. The difference of mechanical free energy density and binding energy between the membrane conformations preferred by the proteins and the lipids reads $\Delta f \equiv \Delta f_{\text {mech }}=\frac{1}{2} \kappa\left(u_{\text {opt }}-u_{0}\right)^{2}$ and $\Delta E \equiv$ $\Delta E_{\mathrm{B}}=\frac{1}{2} \epsilon\left(u_{\mathrm{opt}}-u_{0}\right)^{2}$, respectively.

Upon eliminating the mechanical d.o.f. using $u(m)$, the interplay between chemical and mechanical terms becomes obvious in the dependence of the free energy density on membrane protein density [Fig. 2(a)],

$$
\frac{f}{\Delta f}=\frac{\tilde{m}}{1+\tilde{m}}+\tilde{m}\left(1+\tilde{\gamma} \tilde{m}^{6}\right) \frac{E_{\mathrm{opt}}}{\Delta E}
$$

where $\tilde{m}:=m / m_{\times}$and $\tilde{\gamma}:=\gamma / m_{\times}^{6}$. The first term encodes free energy costs for membrane deformation through protein binding. With increasing protein density $m$, this contribution saturates, as the membrane deforms towards a binding-favorable conformation, implying that the corresponding mechanical free energy costs for binding of additional proteins diminish. For intermediate membrane protein densities, the benefit from protein binding [second term in Eq. (4)] dominates. Finally, for very high protein densities, protein binding becomes unfavorable due to crowding $(\tilde{\gamma}<0)$.

The chemical potential at the membrane, $\mu_{\mathrm{m}}=\partial_{m} f$, i.e., the energy needed to bind one additional protein to the membrane, reads

$$
\frac{\mu_{\mathrm{m}}(\tilde{m})}{E_{\mathrm{opt}}}=1+7 \tilde{\gamma} \tilde{m}^{6}+\frac{\Delta E}{E_{\mathrm{opt}}} \frac{1}{(1+\tilde{m})^{2}} .
$$

In the absence of crowding effects, the chemical potential approaches the optimal value $E_{\mathrm{opt}}<0$ for large protein densities on the membrane, $m \gg m_{\times}$, meaning that there is an energy gain upon binding [Fig. 2(b), dashed lines]. Crowding counteracts this gain, such that protein binding at
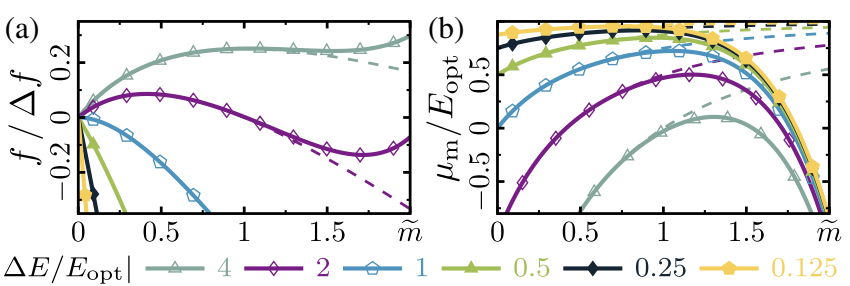

FIG. 2. (a) Free energy density $f / \Delta f$ and (b) membrane chemical potential $\mu_{\mathrm{m}} / E_{\text {opt }}$ plotted as a function of the density of membrane-bound proteins, $m / m_{\times}$, for a series of different protein binding specificities, $\left|\Delta E / E_{\text {opt }}\right|$, indicated in the graph. Solid lines represent $\tilde{\gamma}=-0.004$; dashed lines represent a system without crowding effects, $\tilde{\gamma}=0$.

high densities becomes unfavorable [Fig. 2(b), solid lines]. For low densities $\left(m<m_{\times}\right)$, protein binding is also disfavored, as there is a free energy cost for mechanically deforming the membrane that is largest for low membrane protein densities $m$; cf. the last term in Eq. (5). The amplitude of this reduction is given by $\left|\Delta E / E_{\text {opt }}\right|$, which we term the protein binding specificity, as proteins with a higher specificity have a greater preference for mechanical states other than the relaxed state of the membrane [Fig. 2(b)]. The less specific the binding of a protein, the smaller the changes in the chemical potential as a function of the protein density on the membrane.

What, then, are the implications of these thermodynamic considerations for the kinetics of protein binding and detachment? To answer this question, one has to solve a first-passage-time problem for a particle diffusing in a chemical potential as described by the Smoluchowski equation (1). This is a well-studied problem, which dates back to Kramers' theory of reaction kinetics [74]. For a one-dimensional reaction coordinate $x$, with a reflective boundary at $x=a$ and an absorbing boundary at $x=b$, the first-passage time is given by $[55,56]$

$$
\tau=\frac{1}{D} \int_{a}^{b} d x e^{+\mu(x) / k_{\mathrm{B}} T} \int_{a}^{x} d y e^{-\mu(y) / k_{\mathrm{B}} T},
$$

where $\mu(x)$ is the spatial profile of the chemical potential. In Kramers' classical escape problem, the reaction rate depends on the height of the barrier that the particle has to cross by diffusion to reach its target [74]. In our case, however, there is no such barrier. Instead, as discussed above, we expect the landscape to exhibit a monotonically increasing or decreasing profile, depending on whether the chemical potential at the membrane, $\mu_{\mathrm{m}}$, is larger or smaller than the value in the bulk of the cytosol $\left(\mu_{\text {cyt }}=0\right)$; for an illustration, see Fig. 1.

To estimate the kinetic rates, we simplify the geometry of the cell as follows. We divide the space near the membrane into small reaction compartments with respective sizes given by the average distance $\xi$ between proteins, such that each compartment contains a single protein on average. 
Then, one may approximate a binding process as a one-dimensional diffusion process: An initially unbound protein diffusing in the cytosol enters one of these compartments at a distance $\xi$ from the membrane and, after some time, encounters the membrane located at $x=0$. To calculate the corresponding first-passage time, the membrane is considered as an absorbing boundary. The cytosolic boundary of each compartment can effectively be approximated as a reflective boundary since (on average) there is always one protein within each compartment; i.e., a protein leaving the compartment at $x=\xi$ is replaced by one entering the compartment. Similarly, an unbinding process may be idealized as a stochastic process, where an initially bound protein detaches at $x=0$ (reflective boundary) and leaves the compartment at $x=\xi$ (absorbing boundary).

Given our limited knowledge of the profile of the chemical potential, we chose to approximate it by a piecewise linear function [Fig. 1(a)]. The protein diffuses freely $(\mu=0)$ at large distances from the membrane $(x>d)$. In the vicinity of the membrane $(x<d)$, we assume a linear profile $\mu=\mu_{\mathrm{m}}(1-x / d)$. In the following, we discuss-for simplicity-the case where $\xi=d$. The more general (and more realistic) case, where the protein also crosses a preceding flat potential of length $\xi-d>0$, yields qualitatively similar results and is discussed in the SM [66]. With these approximations, we can use Eq. (6) to obtain an explicit analytic expression for the mean firstpassage times $\tau_{ \pm}$of attachment and detachment [75]. The corresponding kinetic rates, $k_{ \pm}=\tau_{ \pm}^{-1}$, expressed in units of the basic diffusion time $\tau:=2 D / \xi^{2}$, are found to be

$$
k_{ \pm} \tau=\frac{1}{2}\left(\frac{\mu_{\mathrm{m}}}{k_{\mathrm{B}} T}\right)^{2}\left(e^{ \pm \mu_{\mathrm{m}} / k_{\mathrm{B}} T} \mp \frac{\mu_{\mathrm{m}}}{k_{\mathrm{B}} T}-1\right)^{-1}
$$

These rates exhibit a pronounced nonlinear dependence on the membrane protein density (Fig. 3). Hence, protein attachment and detachment are both cooperative processes, owing to the mechanochemical coupling mediated by membrane elasticity. By fitting the attachment rate, Eq. (7), at low densities with $k_{+} \approx a+b \tilde{m}^{n}$, we infer a relationship between the protein specificity $\left|\Delta E / E_{\text {opt }}\right|$ and the (Hill) cooperativity coefficient $n$ [Figs. 3(c) and 3(d)]; for an analysis in terms of Hill curves, refer to the SM [66]. Strong cooperativity $(n>1)$ occurs only for high protein specificities, $\left|\Delta E / E_{\mathrm{opt}}\right|>1$. This implies that induction of a membrane conformation that favors protein binding requires the binding of a disproportionally large number of proteins to the membrane. Therefore, in the deterministic limit, proteins would not attach to the membrane at all [Fig. 2(a), empty triangles and diamonds]. However, stochastic binding events, while unlikely at low protein densities, reduce the free energy cost of subsequent binding events and thereby increase their likelihood. This positive feedback leading to recruitment is a purely stochastic effect
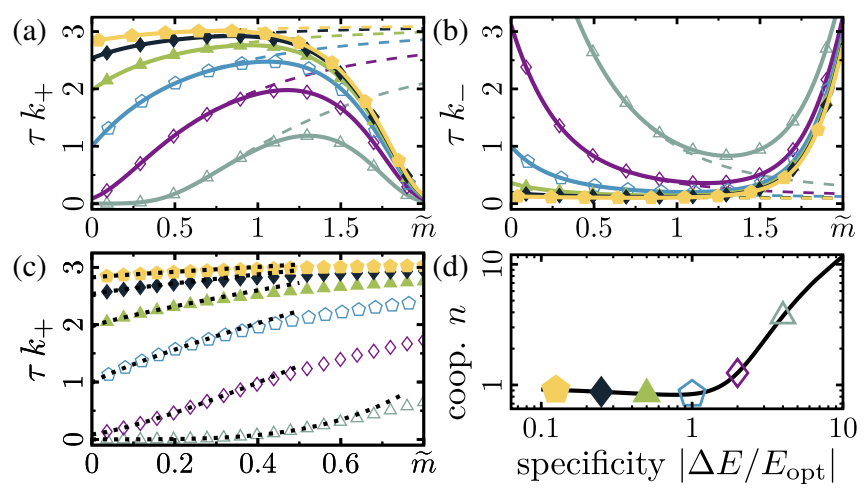

FIG. 3. Kinetic rates for membrane attachment $k_{+} \tau$ (a) and detachment $k_{-} \tau(\mathrm{b})$ as a function of $\tilde{m}$ for $E_{\mathrm{opt}}=-5 k_{\mathrm{B}} T$ and a set of protein specificities $\left|\Delta E / E_{\text {opt }}\right|$ as in Fig. 2. Solid and dashed lines represent $\tilde{\gamma}=-0.004$ and $\tilde{\gamma}=0$, respectively. (c) For low membrane protein concentrations $\tilde{m}$, the attachment rate can be approximated by $a+b \tilde{m}^{n}$; corresponding fits are indicated by the dotted lines. (d) The cooperativity coefficient $n$ increases with protein specificity $\left|\Delta E / E_{\text {opt }}\right|$.

and is related to nucleation during discontinuous phase transitions.

To assess whether the proposed indirect cooperativity mechanism could actually come into play at physiological protein concentrations, we estimate its various parameters from known literature values. For proteins with a membrane curvature sensing domain, typical values for the optimal curvature and binding energy are $H_{\text {opt }}=0.1 \mathrm{~nm}^{-1}$ $[21,76]$ and $E_{\mathrm{opt}} \approx-5 k_{\mathrm{B}} T$ [77]; we assume vanishing spontaneous curvature $\left(H_{0}=0\right)$. Across different studies, the bending modulus of a phospholipid bilayer was measured to be in the range of $\kappa \approx 10 \ldots 50 k_{\mathrm{B}} T$, suggesting a typical value $\kappa \approx 30 k_{\mathrm{B}} T[28,78,79]$. Taking a value $\left|\Delta E / E_{\mathrm{opt}}\right|=2$ for protein specificity where nonlinear binding kinetics is significant (recruitment) [Fig. 3(d)], the corresponding range of concentrations, $m<m_{\times} \approx$ $3 \times 10^{4} \mu \mathrm{m}^{-2}$, easily encompasses any physiological value; the maximum packing density of proteins with size $10 \mathrm{~nm}$ is $1 \times 10^{4} \mu \mathrm{m}^{-2}$.

In summary, we have shown that mechanochemical coupling between proteins provides a possible mechanism for the nonlinear binding kinetics (recruitment) of proteins to the membrane. The effect originates from the interplay between protein-lipid and lipid-lipid interactions, which induce mechanical deformations of the membrane and thereby alter the protein binding environment. As protein-lipid interactions become dominant with increasing concentrations of membrane-bound proteins, the membrane's mechanical state becomes more favorable for binding. This shows how cooperativity and the recruitment of proteins can naturally emerge without any reliance on direct chemical interactions and conformational changes. The results should certainly be applicable to proteins that are known to bend membranes, e.g., proteins containing 
BAR domains [20-23,26]. As recent experiments have unexpectedly shown that Min protein oscillations can lead to oscillations in vesicle shape [27], we would argue that our theory should also apply to the broad class of NTPases that are essential for cellular protein pattern formation. Thus, strain sensing and generation might not only be a property of a few specialized proteins but might actually be a prominent and perhaps general feature of membranebinding proteins. Further exploration of curvature sensing during macroscopic pattern formation might be highly rewarding [80-82]. Our theory predicts that one can alter the recruitment exponent $n$ of membrane-binding proteins by tuning the protein specificity (possibly by changing the membrane composition or introducing permanently bound membrane-bending proteins). Such a change in cooperativity should have a much stronger effect on emerging protein patterns than the tuning of reaction rates because it changes the nature of the nonlinear coupling. We would expect profound changes in the protein dynamics that could be explored using appropriately modified reaction-diffusion models for various cellular systems [83-88], as well as by experimentally tinkering with the composition of the membrane. Finally, it would be highly interesting and rewarding to quantify the mechanochemical effect for specific membrane-binding proteins experimentally. This would provide an interesting basis for theoretical models of pattern-forming protein systems and contribute towards revealing the universal role of membrane elasticity in cellular functions.

We thank Fridtjof Brauns, George Dadunashvili, Raphaela Geßele, Igor Goychuk, Isabella Graf, Laeschkir Würthner, Timon Idema, Anatoly B. Kolomeisky, Thomas Litschel, Rüdiger Thul, and Manon Wigbers for stimulating discussions. E. F. acknowledges financial support from the Deutsche Forschungsgemeinschaft (DFG) via the Collaborative Research Center (SFB) 1032 (project B2). A. G. is supported by the DFG through the Graduate School of Quantitative Biosciences Munich (QBM). E. F. also acknowledges the hospitality of the Kavli Institute of Nanoscience at TU Delft, where part of this work was done.

* Corresponding author.

frey@lmu.de

[1] J. Halatek, F. Brauns, and E. Frey, Phil. Trans. R. Soc. B 373, 20170107 (2018).

[2] J. Lutkenhaus, Annu. Rev. Biochem. 76, 539 (2007).

[3] D. I. Johnson, Microbiol. Mol. Biol. Rev. 63, 54 (1999).

[4] B. Goldstein and I. G. Macara, Dev. Cell 13, 609 (2007).

[5] C. D. Lawson and A. J. Ridley, J. Cell Biol. 217, 447 (2018).

[6] A. V. Hill, Biochem. J. 7, 471 (1913).

[7] M. I. Stefan and N. Le Novère, PLoS Comput. Biol. 9, e1003106 (2013).

[8] E. Fischer-Friedrich and N. Gov, Phys. Biol. 8, 026007 (2011).
[9] M. Encinar, A. V. Kralicek, A. Martos, M. Krupka, S. Cid, A. Alonso, A. I. Rico, M. Jiménez, and M. Vélez, Langmuir 29, 9436 (2013).

[10] M. G. J. Ford, I. G. Mills, B. J. Peter, Y. Vallis, G. J. K. Praefcke, P. R. Evans, and H. T. McMahon, Nature (London) 419, 361 (2002).

[11] I. Tsafrir, Y. Caspi, M.-A. Guedeau-Boudeville, T. Arzi, and J. Stavans, Phys. Rev. Lett. 91, 138102 (2003).

[12] M. C. Lee, L. Orci, S. Hamamoto, E. Futai, M. Ravazzola, and R. Schekman, Cell 122, 605 (2005).

[13] N. S. Gov and A. Gopinathan, Biophys. J. 90, 454 (2006).

[14] J. Zimmerberg and M. M. Kozlov, Nat. Rev. Mol. Cell Biol. 7, 9 (2006).

[15] W. A. Prinz and J. E. Hinshaw, Crit. Rev. Biochem. Mol. Biol. 44, 278 (2009).

[16] J. C. Stachowiak, E. M. Schmid, C. J. Ryan, H. S. Ann, D. Y. Sasaki, M. B. Sherman, P. L. Geissler, D. A. Fletcher, and C. C. Hayden, Nat. Cell Biol. 14, 944 (2012).

[17] H. T. McMahon and E. Boucrot, J. Cell Sci. 128, 1065 (2015).

[18] I. K. Jarsch, F. Daste, and J. L. Gallop, J. Cell Biol. 214, 375 (2016).

[19] N. S. Gov, Phil. Trans. R. Soc. B 373, 20170115 (2018).

[20] J. Zimmerberg and S. McLaughlin, Curr. Biol. 14, R250 (2004).

[21] B. J. Peter, H. M. Kent, I. G. Mills, Y. Vallis, P. J. G. Butler, P. R. Evans, and H. T. McMahon, Science 303, 495 (2004).

[22] V. K. Bhatia, K. L. Madsen, P.-Y. Bolinger, A. Kunding, P. Hedegård, U. Gether, and D. Stamou, EMBO J. 28, 3303 (2009).

[23] C. Mim and V. M. Unger, Trends Biochem. Sci. 37, 526 (2012).

[24] C. Zhu, S. L. Das, and T. Baumgart, Biophys. J. 102, 1837 (2012).

[25] C. Prévost, H. Zhao, J. Manzi, E. Lemichez, P. Lappalainen, A. Callan-Jones, and P. Bassereau, Nat. Commun. 6, 8529 (2015).

[26] M. Simunovic, G. A. Voth, A. Callan-Jones, and P. Bassereau, Trends Cell Biol. 25, 780 (2015).

[27] T. Litschel, B. Ramm, R. Maas, M. Heymann, and P. Schwille, Angew. Chem. Int. Ed. 57, 16286 (2018).

[28] R. Phillips, T. Ursell, P. Wiggins, and P. Sens, Nature (London) 459, 379 (2009).

[29] T. R. Weikl, Annu. Rev. Phys. Chem. 69, 521 (2018).

[30] T. Idema and D. J. Kraft, Curr. Opin. Colloid Interface Sci. 40, 58 (2019).

[31] H. Huang, Biophys. J. 50, 1061 (1986).

[32] P. Wiggins and R. Phillips, Biophys. J. 88, 880 (2005).

[33] O. S. Andersen and R.E. Koeppe, Annu. Rev. Biophys. Biomol. Struct. 36, 107 (2007).

[34] D. Milovanovic, A. Honigmann, S. Koike, F. Göttfert, G. Pähler, M. Junius, S. Müllar, U. Diederichsen, A. Janshoff, H. Grubmüller, H. J. Risselada, C. Eggeling, S. W. Hell, G. van den Bogaart, and R. Jahn, Nat. Commun. 6, 5984 (2015).

[35] A. Grau-Campistany, E. Strandberg, P. Wadhwani, J. Reichert, J. Bürck, F. Rabanal, and A.S. Ulrich, Sci. Rep. 5, 9388 (2015).

[36] M. S. Turner and P. Sens, Phys. Rev. Lett. 93, 118103 (2004). 
[37] A. Iglič, T. Slivnik, and V. Kralj-Iglič, J. Biomech. 40, 2492 (2007).

[38] R. Shlomovitz and N. S. Gov, Phys. Biol. 6, 046017 (2009).

[39] Ś. Perutková, V. Kralj-Iglič, M. Frank, and A. Iglič, J. Biomech. 43, 1612 (2010).

[40] L. Mesarec, W. Góźdź, V. K. Iglič, S. Kralj, and A. Iglič, Colloids Surf. B 141, 132 (2016).

[41] J. Agudo-Canalejo and R. Lipowsky, Soft Matter 13, 2155 (2017).

[42] M. Goulian, R. Bruinsma, and P. Pincus, Europhys. Lett. 22, 145 (1993).

[43] R. Golestanian, M. Goulian, and M. Kardar, Europhys. Lett. 33, 241 (1996).

[44] L. V. Schäfer, D. H. de Jong, A. Holt, A. J. Rzepiela, A. H. de Vries, B. Poolman, J. A. Killian, and S. J. Marrink, Proc. Natl. Acad. Sci. U.S.A. 108, 1343 (2011).

[45] L. D. Renner and D. B. Weibel, J. Biol. Chem. 287, 38835 (2012).

[46] V. Corradi, E. Mendez-Villuendas, H. I. Ingólfsson, R.-X. Gu, I. Siuda, M. N. Melo, A. Moussatova, L. J. DeGagné, B. I. Sejdiu, G. Singh, T. A. Wassenaar, K. D. Magnero, S. J. Marrink, and D. P. Tieleman, ACS Cent. Sci. 4, 709 (2018).

[47] C. A. Haselwandter and R. Phillips, Europhys. Lett. 101, 68002 (2013)

[48] Y. Schweitzer and M. M. Kozlov, PLoS Comput. Biol. 11, e1004054 (2015).

[49] C. van der Wel, A. Vahid, A. Šarić, T. Idema, D. Heinrich, and D. J. Kraft, Sci. Rep. 6, 32825 (2016).

[50] A. Vahid and T. Idema, Phys. Rev. Lett. 117, 138102 (2016).

[51] U. Schmidt, G. Guigas, and M. Weiss, Phys. Rev. Lett. 101, 128104 (2008).

[52] C. A. Haselwandter and N. S. Wingreen, PLoS Comput. Biol. 10, e1003932 (2014).

[53] A. Vahid, A. Šarić, and T. Idema, Soft Matter 13, 4924 (2017).

[54] A. Vahid and T. Idema, bioRxiv https://doi.org/10.1101/336545 (2018).

[55] C. Gardiner, Stochastic Methods (Springer, Berlin, 2009).

[56] R. Zwanzig, Nonequilibrium Statistical Mechanics (Oxford University Press, Oxford, 2001).

[57] In general, note that this implies that the chemical potential is a function of cytosolic position $\mathbf{x}$ and a functional of membrane protein density, $m(\boldsymbol{\sigma})$.

[58] W. Helfrich, Z. Naturforsch. C 28C, 693 (1973).

[59] U. Seifert, Adv. Phys. 46, 13 (1997).

[60] We further relate our approach to Helfrich's formulation of the bending energy cost [58] in the SM [66].

[61] Note that the entropic effects of a large protein density can also reduce the protein binding energy, as discussed in the SM [66]. There, we show that the general result of nonlinear protein recruitment to the membrane remains valid.

[62] W. F. Zeno, U. Baul, W. T. Snead, A. C. M. DeGroot, L. Wang, E. M. Lafer, D. Thirumalai, and J. C. Stachowiak, Nat. Commun. 9, 4152 (2018).
[63] V. Kralj-Iglič, V. Heinrich, S. Svetina, and B. Žekš, Eur. Phys. J. B 10, 5 (1999).

[64] R. Shlomovitz, N. S. Gov, and A. Roux, New J. Phys. 13, 065008 (2011).

[65] B. Božič, S. L. Das, and S. Svetina, Soft Matter 11, 2479 (2015).

[66] See Supplemental Material at http://link.aps.org/ supplemental/10.1103/PhysRevLett.123.178101 for further details and an additional analysis of the model, which includes Refs. [67-72].

[67] O.-Y. Zhong-can and W. Helfrich, Phys. Rev. A 39, 5280 (1989).

[68] B. Božič, V. Kralj-Iglič, and S. Svetina, Phys. Rev. E73, 041915 (2006).

[69] M. Doi, Soft Matter Physics (Oxford University Press, Oxford, 2013).

[70] M. Deserno, Chem. Phys. Lipids 185, 11 (2015).

[71] A. Guckenberger and S. Gekle, J. Phys. Condens. Matter 29, 203001 (2017).

[72] A. Goychuk, L. Würthner, M. Wigbers, and E. Frey (to be published).

[73] A further generalization yielding normal and tangential stresses involves variational surface calculus and is briefly outlined in the SM [66]. There, we show that the analysis presented here is valid in the limit of small deformations.

[74] H. A. Kramers, Physica 7, 284 (1940).

[75] S. Bell and E. M. Terentjev, Biophys. J. 112, 2439 (2017).

[76] W. Wu, K.-T. Park, T. Holyoak, and J. Lutkenhaus, Mol. Microbiol. 79, 1515 (2011).

[77] L. Ma, Y. Cai, Y. Li, J. Jiao, Z. Wu, B. O'Shaughnessy, P. De Camilli, E. Karatekin, and Y. Zhang, eLife 6, e30493 (2017).

[78] R. Dimova, Adv. Colloid Interface Sci. 208, 225 (2014).

[79] J. F. Nagle, M. S. Jablin, S. Tristram-Nagle, and K. Akabori, Chem. Phys. Lipids 185, 3 (2015).

[80] B. Peleg, A. Disanza, G. Scita, and N. Gov, PLoS One 6, e18635 (2011).

[81] D. Thalmeier, J. Halatek, and E. Frey, Proc. Natl. Acad. Sci. U.S.A. 113, 548 (2016).

[82] Z. Wu, M. Su, C. Tong, M. Wu, and J. Liu, Nat. Commun. 9, 136 (2018).

[83] K. C. Huang, Y. Meir, and N. S. Wingreen, Proc. Natl. Acad. Sci. U.S.A. 100, 12724 (2003).

[84] J. Halatek and E. Frey, Cell Rep. 1, 741 (2012).

[85] B. Klünder, T. Freisinger, R. Wedlich-Söldner, and E. Frey, PLoS Comput. Biol. 9, e1003396 (2013).

[86] J. Denk, S. Kretschmer, J. Halatek, C. Hartl, P. Schwille, and E. Frey, Proc. Natl. Acad. Sci. U.S.A. 115, 4553 (2018).

[87] A. B. Goryachev and M. Leda, Mol. Biol. Cell 28, 370 (2017).

[88] J. Halatek and E. Frey, Nat. Phys. 14, 507 (2018). 